\title{
Therapeutical Effect of Extracts of Terminalia chebula In Inhibiting Human Pathogens and Free Radicals
}

\author{
Dolly Singh, Deepti Singh, Soon Mo Choi, Sun Mi Zo, Saet Byul Ki, and Sung Soo Han
}

\begin{abstract}
In recent years, multiple drug resistance has been developed due to indiscriminate use of existing antimicrobial drugs in the treatment of infectious diseases. Conventional antibiotics are strong medicines, which if not used in precise way may cause harmful effects. The major thrust is to establish alternative antimicrobial agent in order to treat microbial infections with less or no toxic effect to body and less negligible side effects. The herbal medicines have shown potential to overcome the limitation associated with conventional drugs. However, the appropriate choice of source for herbal medicine is also very important. Terminalia chebula possesses potential pharmaceutical activities and used in several Ayurvedic formulations. The study of solvent-free organic (ethylacetate, acetone, methanol of increasing polarity) and aqueous extracts of the fruits of $T$. chebula, showed the potential to reduce growth of microorganisms, minimizing the risk of infection, while optimizing the conditions to encourage healing. In our study we found, methanol extract as a potential bactericidal and potent antioxidant while aqueous extract showed the least potential as an antimicrobial agent, though a moderate antioxidant. The finding may provide scientific rationale for the use of crude extract of the plant as a new drug compound as potential antioxidant and antimicrobial agent.
\end{abstract}

Index Terms-Antioxidant, Ayurvedic, Bactericidal, Terminalia chebula.

\section{INTRODUCTION}

Many infectious diseases like meningitis, bacteremia, pneumonia, scalded skin syndrome etc are caused by most commonly yet pathogenic bacteria belonging to Streptococcus and Staphylococcus genera [1]-[5]. The resistance of these microorganisms due to excessive use of antibiotics over the years has led to the development of more aggressive and pathogenic strains of them. There is an urgent need for development of effective therapeutics against these resistant strains. Traditional medicinal plants have many therapeutic properties, are accessible by poor community of the world and are economically effective means of treatments for many deadly diseases [6]. Several plant extracts has been tested for their anti-microbial properties world-over [6]-[8].

Manuscript received April 25, 2012; revised May 31, 2012. This work was supported by grant No. RTI04-01-04 from the Regional Technology Innovation Program of the Ministry of Knowledge

Sung Soo Han is with the Dept of Nano, Medical, and Polymer Materials Yeungnam University 214-1 Daedong, Gyeongsan 712749, Korea . (Tel.: +82-53-810-2773, fax: +82-53-810-4685 e-mail:sshan@yu.ac.kr)

Dolly Singh, Deepti Singh, Soon Mo Choi, Sun Mi Zo, Saet Byul Ki are with Dept of Nano, Medical, and Polymer Materials Yeungnam University 214-1 Daedong, Gyeongsan 712749, Korea (e-mail: dollys@yu.ac.kr, deeptis@yu.ac.kr, debate311@nate.com, koy41@nate.com, 87ksb@hanmail.net).
Terminalia chebula is used as a mild laxative and as an astringent against wounds and abscesses. Practictioners of folk medicine in India and Southeast Asia use the fruit for homeostatic, laxative and as cardiotonic. It is used as a remedy against a sore throat and cough, against diarrhoea connected with a prolapsed rectum in China and against ulcers and dysentery in Tibet. The antibacterial activity of various extracts of Chebula myrobalan powder was tested against Streptococcus species, Staphylococcus aureus and Pseudomonas aeruginosa.

\section{MATERIALS}

T. chebula powder was purchased from local market of Mumbai, India. Pathogens of MTCC grade were obtained from IMTECH (Chandigarh, India). Gallic acid and Glutaraldehyde was purchased from s.d. fine-chemicals limited (Mumbai, India). Gentamicin and 2,4,6-tripyidyl-s-triazine (TPTZ) were procured from Hi-Media(Mumbai,India), 3-(4,5-dimethylthiazol-2-yl)-2,5diphenyl tetrazolium bromide (MTT, 98\%) reagent, 4'-6-diamidino-2-phenylindole (DAPI), propidium iodide (PI) and nystatin was purchased from Sigma (St. Louis, USA). Fetal bovine serum (FBS) and streptomycin-penicillin antibiotic solutions were bought from HyClone (Utah, USA). All other chemicals used were of analytical grade. SIRC (rabbit corneal epithelial cells) cell line was obtained from NCL, Pune, India

\section{METHODS}

Preparation of $\boldsymbol{T}$. chebula extracts: $25 \mathrm{~g}$ of powder of fruits of T.chebula was taken in a thimble. Extracts were prepared in the series of $500 \mathrm{ml}$ of different solvents based on increasing polarity (Ethyl acetate, Acetone, Methanol, and Water) using Soxhlet extraction method. The soxhletting cycle was completed after the solvents turned colorless in the tube indicating the complete extraction of the phytosignatures. The solvents were evaporated to dryness using rotary evaporator and the extract obtained was lyophilized at $-50{ }^{\circ} \mathrm{C}$. The lyophilized solvent-free extracted powder was stored in airtight bottles at $4{ }^{\circ} \mathrm{C}$ till further experimentation. [9].

\section{A. Determination of Antimicrobial Activity of T.chebula Extracts}

Antibacterial activities of all the solvent free extracts of T.chebula (ethyl acetate S1, acetone S2, methanol S3 and aqueous-S4) were determined by agar well diffusion method. The extracts were dissolved in DMSO (dimethylsulphoxide). 
The microbial lyophilized cultures were revived at $37^{\circ} \mathrm{C}$ for $18 \mathrm{~h}$ in a broth medium and the culture was adjusted to $5 \times 10^{5}$ $\mathrm{cfu} / \mathrm{ml}$ in accordance with the McFarland Turbidity standards [10]. The $20 \mu 1$ of the culture was spread on Mueller Hinton agar plates and wells of $9 \mathrm{~mm}$ diameter were punched into the agar plates. $100 \mu \mathrm{l}$ of the solvent-free extracts of concentration $0.5 \mathrm{mg} / 100 \mu \mathrm{l}$ and $1 \mathrm{mg} / 100 \mu \mathrm{l}$ were used for determination of ZOI (Zone of Inhibition). After holding the plates at room temperature for 2 hours to allow diffusion of the extract into the media, the plates were incubated at $37^{\circ} \mathrm{C}$ for $24 \mathrm{~h}$. DMSO and Gentamicin was used as a negative control and reference antibiotic (positive control) respectively. The test was performed in triplicates and the final results were presented as the mean zone of inhibition [11]. Inhibition Zone was observed after $24 \mathrm{~h}$ and Zone of inhibition was recorded for all the extracts and gentamicin. Inhibition Zone Diameter (IZD) was measured to the nearest millimeter $(\mathrm{mm})$ by reducing the IZD value with Diameter of the well bored.

Total Zone of Inhibition = Inhibition Zone DiameterDiameter of the well.

\section{B. Determination of Minimal Inhibitory Concentration (MIC)}

The Minimal Inhibitory Concentration (MICs) of the extracts of the plant was determined against the tested bacteria by macro broth dilution assay method [12]. Two-fold serial dilutions of all the extracts (based on their IZD results: $0.5 \mathrm{mg} / 100 \mu \mathrm{l}$ ) were prepared in 24 well plates with MHA as diluents. Each dilution was seeded with $20 \mu 1$ of test microorganisms to the standard concentration $\left(5 \times 10^{5} \mathrm{cfu} / \mathrm{ml}\right)$. Two-fold serial dilution of Gentamicin was used as experimental positive control. The plates were incubated at $37^{\circ} \mathrm{C}$ for $24 \mathrm{~h}$. The least concentration of the extract or standard drug showing no visible growth was taken as the MIC. After $24 \mathrm{~h}$ of incubation period, mean MIC values were calculated. The test was performed in triplicates for each microorganism used and the final results were expressed as the arithmetic average of triplicate experiments. $20 \mu 1$ test media from each MIC broth tube was spread over the MHA plates. Plates were incubated at $37^{\circ} \mathrm{C}$ for $24 \mathrm{~h}$. The test MIC concentration showing no bacterial growth on agar plates was considered as Minimum Bactericidal Concentration (MBC) of the extract [11].

\section{Determination of Antioxidant Activity of T. chebula Extracts}

The ferric-reducing antioxidant power (FRAP) assay measures the antioxidant potentials of "antioxidants" to reduce the $\mathrm{Fe} 3+/$ 2,4,6-tripyidyl-s-triazine (TPTZ) complex present in stoichiometric excess to the blue colored Fe2+-TPTZ form. (22). The stock solution of various extracts of concentration $0.3 \mathrm{mgml}^{-1}$ was prepared. $10 \mu 1-100 \mu 1(6 \mathrm{X}$ $10-4 \mathrm{~g} / \mathrm{l}-60 \mathrm{X} 10-4 \mathrm{~g} / \mathrm{l})$ of extract was mixed with $1.5 \mathrm{ml}$ of FRAP reagent and the volume was adjusted to $5 \mathrm{ml}$ with distilled water. The test tubes containing the test solutions were incubated at $37^{\circ} \mathrm{C}$ for $15 \mathrm{~min}$. The absorbance was recorded at 593nm [13].

\section{REsUlts}

\section{A. Determination of antimicrobial activity of T.chebula extracts}

Among all the extracts of $T$. chebula, S3 was found to be most potential in inhibiting M1 bacterium giving the highest Zone of Inhibition value of $19.33 \mathrm{~mm}$ at $0.5 \mathrm{mg} / 100 \mu \mathrm{l}$ and $21.00 \mathrm{~mm}$ at $1 \mathrm{mg} / 100 \mu 1$. M2 was among the most susceptible of all the microorganisms followed by M4, whose growth was effectively inhibited by all the extracts of this plant among which S1, S3 and S4 was found to be most effective. M3 was found resistant to all the extracts (S1-S4) of the plant. Though $T$. chebula extracts was effective against M1-M4 microbes, M5 was found showing resistant to all the extracts of this plant (Fig 1-4).

\section{B. Determination of Minimal Inhibitory Concentration (MIC) and $M B C$}

MIC and MBC values obtained for the extracts of T.chebula, revealed that $\mathrm{S} 1$ and $\mathrm{S} 2$ extract had the same MIC and MBC values and S3 and S4 extract gave the values at par with each other. MIC for M3 and M5 was not performed as the preliminary antimicrobial test showed no results against this bacterium. The least MIC value for M1 was exhibited by S3 and S4 extracts which was lesser than the positive control. Against M2 bacterium, all the extracts of $T$. chebula gave the same value of $0.125 \mathrm{mg} / \mathrm{ml}$. Whereas, M3 was potentially inhibited by $\mathrm{S} 1$ and $\mathrm{S} 2$ extracts having the MIC value of $0.0625 \mathrm{mg} / \mathrm{ml}$. MIC Index value was again same as other extracts..

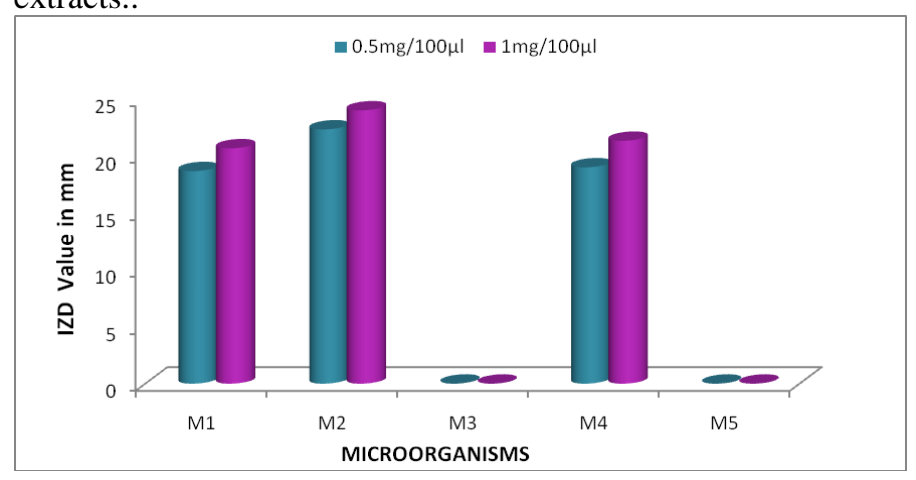

Fig. 1. Demonstration of IZD value (mm) of S1 extract of T. chebula against Streptococcus mutans (M1), Streptococcus pneumonia (M2), Streptococcus pyogenes (M3), Staphylococcus aureus (M5) and Pseudomonas aeruginosa (M5).

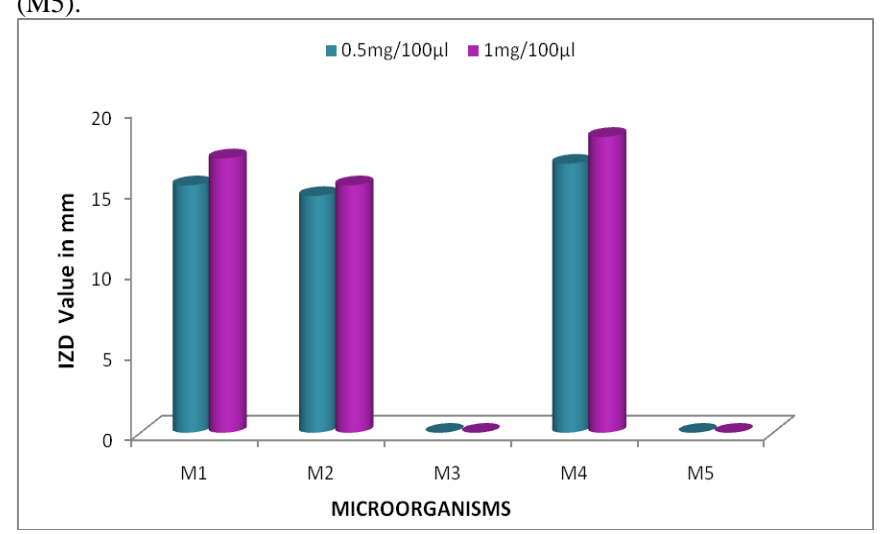

Fig. 2. Demonstration of IZD value (mm) of S2 extract of T. chebula against Streptococcus mutans (M1), Streptococcus pneumonia (M2), Streptococcus pyogenes (M3), Staphylococcus aureus (M5) and Pseudomonas aeruginosa (M5). 


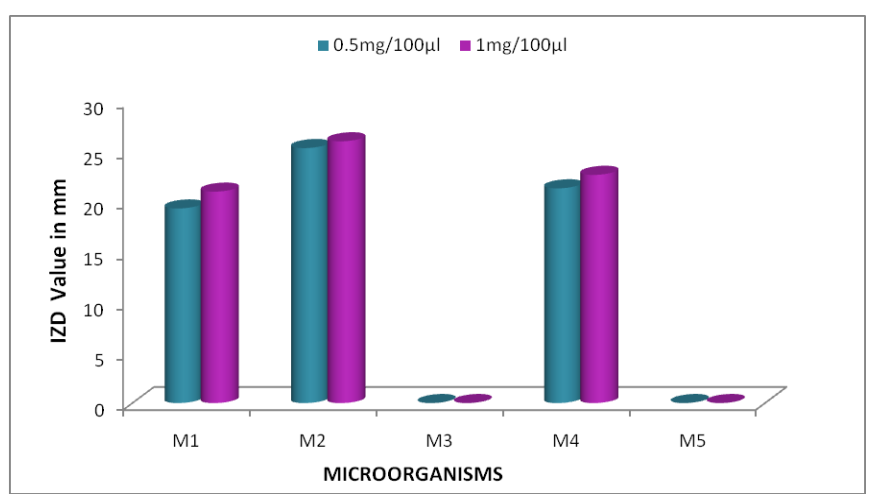

Fig. 3. Demonstration of IZD value ( $\mathrm{mm}$ ) of S3 extract of T. chebula against Streptococcus mutans (M1), Streptococcus pneumonia (M2), Streptococcus pyogenes (M3), Staphylococcus aureus (M5) and Pseudomonas aeruginosa (M5)

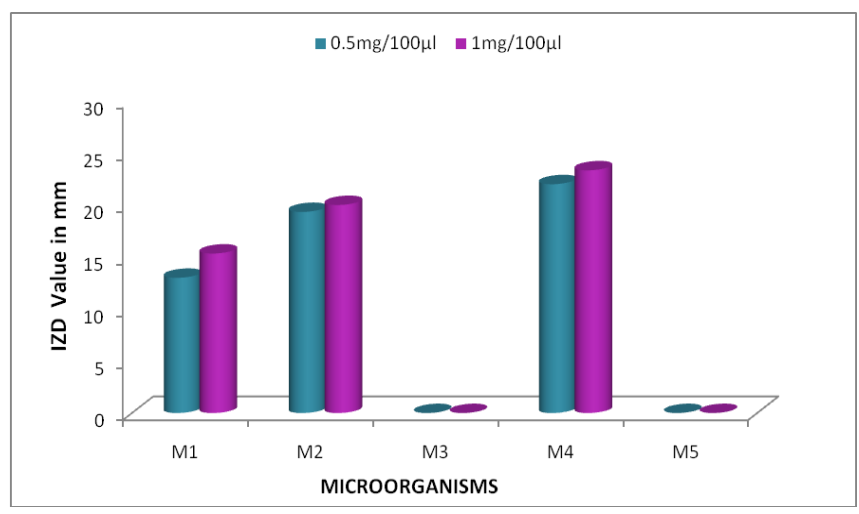

Fig. 4. Demonstration of IZD value (mm) of S4 extract of $T$. chebula against Streptococcus mutans (M1), Streptococcus pneumonia (M2), Streptococcus pyogenes (M3), Staphylococcus aureus (M5) and Pseudomonas aeruginosa (M5).

\section{Determination of Antioxidant Activity of T. chebula Extracts}

TABLE I: REPRESENTS THE ABSORBANCE AND ANTIOXIDANT POWER OF ETHYl ACETATE (S1), ACETONE (S2), Methanol (S3) AND AQueOus (S4) EXTRACT OF T. CHEBULA

\begin{tabular}{|l|l|l|l|l|l|}
\hline S.No & $\begin{array}{c}\text { Concentrati } \\
\text { on of the } \\
\text { extract in g/1 } \\
\text { of S1 }\end{array}$ & $\begin{array}{c}\text { Antioxida } \\
\text { nt Power } \\
\text { extract in } \\
\text { M/1 }\end{array}$ & $\begin{array}{c}\text { Antioxida } \\
\text { nt Power } \\
\text { of S2 } \\
\text { extract in } \\
\text { M/1 }\end{array}$ & $\begin{array}{c}\text { Antioxida } \\
\text { nt Power } \\
\text { of S3 } \\
\text { extract in } \\
\text { M/l }\end{array}$ & $\begin{array}{c}\text { Antioxida } \\
\text { nt Power } \\
\text { of S4 } \\
\text { extract in } \\
\text { M/1 }\end{array}$ \\
\hline 1 & $6 \times 10^{-4}$ & 16.42 & 19.83 & 19.83 & 10.00 \\
\hline 2 & $6 \times 10^{-4}$ & 26.33 & 19.83 & 27.42 & 24.75 \\
\hline 3 & $6 \times 10^{-4}$ & 36.58 & 25.25 & 27.58 & 35.75 \\
\hline 4 & $6 \times 10^{-4}$ & 42.83 & 32.58 & 32.17 & 42.58 \\
\hline 5 & $6 \times 10^{-4}$ & 48.25 & 38.06 & 39.92 & 47.58 \\
\hline 6 & $6 \times 10^{-4}$ & 53.75 & 43.17 & 48.25 & 50.67 \\
\hline 7 & $6 \times 10^{-4}$ & 56.83 & 45.75 & 53.42 & 56.08 \\
\hline 8 & $6 \times 10^{-4}$ & 59.33 & 50.75 & 56.08 & 58.83 \\
\hline 9 & $6 \times 10^{-4}$ & 62.67 & 54.08 & 57.33 & 60.75 \\
\hline 10 & $6 \times 10^{-4}$ & 63.33 & 55.25 & 58.17 & 60.83 \\
\hline
\end{tabular}

The ability of $T$. chebula extracts to scavenge excess of $\mathrm{Fe}^{3+}$ ions or to convert them to more stable ions of $\mathrm{Fe}^{2+}$ exhibited a different result than its other species $T$. bellirica. The free radical scavenging property of the extracts of T.chebula were concentration dependent and showed a trend $\mathrm{S} 1>\mathrm{S} 4>\mathrm{S} 3>\mathrm{S} 2$. At a lower concentration of the extracts S2 and S3 extracts exhibited better scavenging power. With increase in concentration the antioxidant power of S1 and S4 was better than $\mathrm{S} 2$ and $\mathrm{S} 3$, which was increasing with increase in their respective concentration but in comparison was lesser than the S1 and S4 extract. The results are recorded and represented as Table I.

\section{DISCUSSION}

\section{A. Determination of Antimicrobial Property of T. chebula:}

Bag, A. et al., 2009[14] and Chaudhari, M. and Mengi, S., 2005[15] performed the antimicrobial activity on Terminalia species on various microorganisms to obtain the result which was similar to that found in our showing Terminalia species to possess antimicrobial property which also supports data obtained. The present study shows that all the extract of $T$. chebula possessed strain specific antimicrobial activity and S3 extract were found to be the most potential antimicrobial agent in inhibiting M2 microbe, S2 were found to possess the least potential antimicrobial activity giving moderate results as compared to other extracts. Similar results were obtained in case of M1 bacterium where this microbe was found to be susceptible to S3 extract Chebula extracts S1-S4 were ineffective in inhibiting M3 bacterial growth giving no IZD values. This study is comparable to previously reported results in literature where all the four microbes are effectively inhibited with acetone extract of the plants with variations in IZD values [16].

The result of MIC study on T.chebula by Bag, A. et al., 2009 [14], reveals the significant inhibition of uropathogenic strains as well pure strains of pathogens by ethanol and aqueous extracts of Chebulic myrobalans. Our study shows better inhibitory concentrations of having a lower MIC and $\mathrm{MBC}$ values as compared to theirs ' in inhibiting microbes causing various eye infections.

The Ferric-Reducing Antioxidant Power (FRAP) assay measures the antioxidant potentials of antioxidants to reduce the $\mathrm{Fe} 3+/$ 2,4,6-tripyidyl-s-triazine (TPTZ) complex present in stoichiometric excess to the blue coloured $\mathrm{Fe} 2+$ form. The study by various groups of researchers [9], [17] revealed the methanolic, aqueous and chloroform extracts of Chebulic myrobalans to possess antioxidant power to either enhance production of enzymes responsible for scavenging properties or scavenge the oxidants produced during different ailments. The data obtained through our study apparently coincides with the reported ones (though varied methodology was used) wherein the extracts S1-S4 were found to be potent ferric ions reducer, which causes oxidative stress. At even low concentrations of the extracts 6 $\times 10^{-4} \mathrm{~g} / \mathrm{l}$ the antioxidant power of organic extracts like ethyl acetate and acetone were found to possess highest scavenging power as compared to methanolic and aqueous extracts. The antioxidant power was found to be concentration dependent. The free radical scavenging activity in the different extracts decreased in the following order: Ethyl Acetate > Aqueous > Methanol > Acetone. 


\section{CONCLUSION}

The antimicrobial and free radical scavenging study suggests that the plant extracts retained its ability to inhibit the growth of bacteria and antioxidant activities during the course of extraction and can counteract the oxidative damage induced by the pathogens or other means of oxidative stress responsible for various disorders. The phytochemicals of the plants were not compromised as they showed conclusive results in the study suggesting that the extraction procedure applied for obtaining the phytochemicals were optimum. The microbes screened are mostly involved in various pathogenic diseases in humans, S.aureus being the causative agent for bacteremia, and sepsis, S.pneumoniae acute sinusitis, meningitis, bacteremia, sepsis, arthritis, brain abscess, S.pnemoniae and S.pyogenes causes septicaemia and postoperative traumatic etc.

\section{REFERENCES}

[1] M. J. Patterson, "Streptococcus," Baron's Medical Microbiology (Baron S et al., eds.) (4th ed.), Univ of Texas Medical Branch.

[2] K. J. Ryan and C. G. Ray, Sherris Medical Microbiology (4th ed.). McGraw Hill, 2004.

[3] A. C. Siemieniuk, Reed, B. Gregson Dan B, M. Gill John, "The persisting burden of invasive pneumococcal disease in HIV patients: an observational cohort study," BMC Infectious Diseases vol. 11, pp. 314, 2011.

[4] J. Kluytmans, A. Van Belkum, and H. Verbrugh, "Nasal carriage of Staphylococcus aureus: epidemiology, underlying mechanisms, and associated risks," Clin. Microbiol. Rev. vol. 10, no. 3, pp. 505-520, 1997.

[5] A. Clauditz, A. Resch, K. P. Wieland, A. Peschel, F. Götz, "Staphyloxanthin plays a role in the fitness of Staphylococcus aureus and its ability to cope with oxidative stress. Infection and immunity,' vol. 74, no. 8, pp. 4950-4953, 2006.

[6] P. Sumathi and A. Parvathi, "Antimicrobial activity of some traditional medicinal plants," Journal of Medicinal Plants Research, vol. 4, no. 4, pp. 316-321, 2010.
[7] F. Malekzadeh, H. Ehsanifar, M. Shahamat, M. Levin, and R. R Colwell, "Antibacterial activity of black myrobalan (Terminalia chebula Retz) against Helicobacter pylori," International Journal of Antimicrobial Agents vol. 18, pp. 85-88, 2001.

[8] M. M. Golam, R. Mahdia, and K. M. Manjurul, "Antimicrobial Activity of Terminalia chebula," Int, J. Med. Arom. Plants vol. 1, no. 2, pp. 175-179, 2011.

[9] P. Krishnamoorthy, S. Vaithinathan, A. V. Rani, and A. Bhuvaneswari, "Effect of Terminalia chebula fruit extract on lipid peroxidation and antioxidative system of testis of albino rats," African Journal of Biotechnology, vol. 6, no. 16, pp. 1888-1891, 2007.

[10] National Committee for Clinical Laboratory Standards. Performance Standards for antimicrobial disk susceptibility tests- Sixth edition: Approved Standard M2-A6. NCCLS, Wayne, P.A., 1996

[11] D. Singh, N. Chauhan, S. S. Sawhney, and R. M. Painuli, "Biochemical characterization of triphala extracts for developing potential herbal drug formulation for ocular diseases," International Journal of Pharmacy and Pharmaceutical Sciences, vol. 3, no. 5, pp. 516-523, 2011.

[12] National Committee for Clinical Laboratory Standards. Performance Standards for antimicrobial susceptibility testing- eighth informational supplement: Approved Standard M100 S8. NCCLS, Wayne, P.A., 1998.

[13] S. S. Sawhney, R. M. Painuli, and D. Singh, "Antioxidant and antimicrobial activity of phyllanthus emblica for its application in treatment of ophthalmic disorders," Int J Pharm Pharm Sci, vol. 3, no. 4, pp. 129-132, 2011.

[14] A. Bag, S. K. Bhattacharyya, P. Bharati, N. P. Kumar, and R. R. Chattopadhyay, "Evaluation of antimicrobial properties of Chebulic myrobalans (fruit of Terminalia chebula Retz.) extracts against methicillin resistant Staphylococcus aureus and trimethoprim-sulphamethoxazole resistant uropathogenic Escherichia coli," Afr. J. Plant Sci., vol. 3, no. 2, pp. 025-029, 2009.

[15] M. Chaudhari and S. Mengi, "Evaluation of phytoconstituents of Terminalia arjuna for wound healing activity in rats," Phytother Res., vol. 20, no. 9, pp. 799-805.

[16] R. R. Chattopadhyay, S. K. Bhattacharyya, C. Medda, S. Chanda, and A. Bag, "A Comparative Evaluation of Antibacterial Potential of some Plants Used in Indian Traditional Medicine for the Treatment of Microbial Infections," Braz. Arch. Biol. Technol., vol. 52, no. 5, pp. 1123-1128, 2009.

[17] A. Farrukh, A. Iqbal, and M. Zafar, "Antioxidant and free radical scavenging properties of twelve traditionally used Indian medicinal plants," Turk.J.Biol., vol. 30, pp. 177-183, 2006. 